\title{
Verwerfungslinien in Organisationen - die Wirkung von Kontextfaktoren auf die Verbundenheit in Organisationen
}

\author{
Angelika Schmidt ${ }^{1}$ \\ Online publiziert: 5. November 2018 \\ (c) Der/die Autor(en) 2018
}

\section{Zusammenfassung}

Die Bedeutung von Commitment aus Sicht der Unternehmen bzw. der Organisationen wird in den letzten Jahren kontroversiell diskutiert wird. Einerseits wird im Bereich der Personalressourcen ein wesentlicher zentraler Wettbewerbsvorteil gesehen. Gleichzeitig können sehr verschiedene Formen von Beschäftigungsflexibilisierung beobachtet werden. Diese unterschiedlichen Zugänge werden in diesem Beitrag aufgezeigt und die vielseitige Einbettung von Arbeit wie z. B. die Wahrnehmung von Dispositionsmöglichkeiten im Hinblick auf Zeit, auf individuellen Handlungsspielräume oder auch die erlebte soziale Gerechtigkeit präsentiert. All diese Dimensionen bergen das Potenzial in sich, sich als Trenn- bzw. Verwerfungslinien (faultlines) in Organisationen darzustellen und damit auch Einfluss auf die Bindung in Organisationen zu haben.

Schlüsselwörter Organisationales Commitment $\cdot$ Beschäftigungspraktiken $\cdot$ Flexibilisierung

\section{Fault lines in organizations-the effect of contextual factors on the commitment and binding in organizations}

\begin{abstract}
The importance of commitment from the point of view of companies or organizations is discussed controversially in recent years. On the one hand, a key competitive advantage is seen in the field of human resources. At the same time, very different forms of employment flexibilization can be observed. These different approaches are shown in this article and the versatile embedding of work such as the perception of disposition possibilities in terms of time, individual room for maneuver or even the experienced social justice. All of these dimensions have the potential to portray themselves as faultlines in organizations and thus have an impact on the binding in organizations.
\end{abstract}

Keywords Commitment $\cdot$ Employment $\cdot$ Flexibilization

\section{Einleitung}

„We presently are in the midst of what we believe are fundamental changes in the relationship among people, the work they do, and the organization for which they do it" (Oldham und Hackman 2010, S. 466). Diesen Satz prägten Richard Hackman und Greg Oldham, als sie sich mit ihren Model-

Angelika Schmidt

angelika.schmidt@wu.ac.at

1 Department Management, Institut für Change Management und Management Development, Wirtschaftsuniversität Wien, Welthandelsplatz 1, 1020 Wien, Österreich len und Konzepten zur Erklärung von Gruppenprozessen und Teambuilding, wie z. B. dem Job-Characteristics Model erneut auseinandersetzten. Die zu beobachtenden Veränderungen durchziehen verschiedene Ebenen des Handelns und in diesem Beitrag wird aufgezeigt, welche Konsequenzen diese Veränderungen auf der Verbundenheit in und mit Organisationen haben können. Dabei zeigt sich die Verbundenheit in und mit Organisationen in den Spielarten von Mitgliedschaftsregeln, den Ausprägungen von Identifikation und organisationalem Commitment.

Veränderungen lassen sich mannigfach vermerken: Auf den Arbeitsmärkten in den industrialisierten Staaten verschieben sich die Zusammensetzungen der Arbeitskräftepotentiale. In den Unternehmen lassen sich ebenfalls ver- 
schiedene Trends im Hinblick auf den Umgang mit Arbeitskräften beobachten. Parallel dazu gibt es immer mehr empirische Hinweise, dass sich bei jüngeren Generationen beruflichen Zielsetzungen und Prioritäten ändern.

Starten wir mit den aktuellen Befunden einer der bekanntesten Studien zum erlebten Arbeitsumfeld: Seit 2001 werden vom Gallup-Institut in mehreren Industrieländern der Engagement-Index ermittelt. Bei dieser Befragung werden jährlich in einer repräsentativen Stichprobe Arbeitnehmer_innen zu Ihrer Lebens- und Arbeitszufriedenheit befragt. Die Befunde für die D-A-CH Staaten zeigen den Befund, dass in den letzten vier Jahren sich zw. 12-15\% der Arbeitnehmer_innen emotional hoch an ihren jeweiligen Arbeitgeber gebunden fühlen. In ähnlichen Größenordnungen bewegt sich der Anteil derer, die innerlich gekündigt haben - die sich also überhaupt nicht mehr mit dem Unternehmen verbunden fühlen. Dieser Wert liegt in den D-A-CH-Staaten zwischen 12-18\% - ähnlich wie in den USA (Gallup 2017). Diese Engagement-Werte sind seit dem Start der jährlichen Erhebungen ständig gesunken und pendeln sich jetzt seit den letzten fünf Jahren in diesen Größenordnungen ein. Übrig bleibt eine große Gruppe an Arbeitnehmer_innen mit geringer Bindung an ihre Unternehmen und diese schwache Bindung hat Auswirkungen auf das gezeigte Leistungsverhalten.

Interessant ist entsprechend der Blick auf mögliche Erklärungen für diese Entwicklung und wie in vielen Bereichen sind diese mannigfaltig. Zentral herausgehoben werden die sich verändernden Erwartungen der Beschäftigten. So werden in den vertiefenden Interviews im Rahmen dieser jährlichen Erhebung des Indexes von Millenials bedeutsame Arbeit, die Entwicklungspotentiale eröffnet und sich auf die Stärken des/der Einzelnen konzentrieren, als die zentralen Ideen und Erwartungen an Arbeit genannt (Lee et al. 2018). Gleich danach wird darauf verwiesen, dass Arbeit nicht der einzige Fokus sei, sondern es auch noch ein anderes Leben gebe. Diese Ergebnisse zeigen den Wandel der Haltung gegenüber Arbeit. So wird über verschiedenen Qualifikationsstufen hinweg nicht mehr Gehalt und Aufstieg als zentral angesehen, sondern persönliche Entwicklung und Sinnstiftung rücken ins Zentrum, warum Beschäftigte bei einem Unternehmen bleiben oder nach neuen Optionen suchen. Diese Fokusierung geht mit einer erhöhten Bereitschaft einher, Jobs zu wechseln.

Diese Entwicklungen haben Einfluss auf die erlebte Verbundenheit mit dem Unternehmen bzw. der Organisation also dem organisationalen Commitment und stellen personalpolitischen Handlungsoptionen in den Unternehmen vor neue Herausforderungen. Wie kommt es zu diesen Veränderungen und was bedeuten sie?

\section{Bindung als strategischem Wettbewerbsvorteil?}

Die Bedeutung von Bindung ist im weitesten Sinne vom Bedürfnis nach einer Art „situated sense of entity“ (Albert et al. 2000, S. 13) abzuleiten, wobei diese Bedeutung von Commitment aus Sicht der Unternehmen bzw. der Organisationen in den letzten Jahren durchaus kontroversiell diskutiert wurde und wird - das zeigen auch unterschiedlichen Herangehensweisen, die dazu in Organisationen beobachtbar sind: Wir finden einmal den Trend zu High Commitment Management (Harley 2005), High Involvement Management (Appelbaum et al. 2005) oder Psychological Capital Management (Luthans und Youssef 2004), bei dem es darum geht, unverwechselbare und kaum zu imitierende Kapitalarten entstehen zu lassen. So eine Förderung von psychologischem Kapital bringt immer eine langfristige Ausrichtung mit sich, geht aber nachweislich mit strategische Wettbewerbsvorteile (z.B. Sharkie 2005) einher. Gleichzeitig ist in den letzten Jahrzehnten auch eine Ausdifferenzierung von unterschiedlichen institutionalisierten Formen von Arbeit zu beobachten - die oft mit Formen der Entgrenzung von Arbeitsverhältnissen einhergeht.

Viele unserer Betrachtungsweisen basieren auf den Erfahrungen der 1950er-Jahre, in dem sich das Bild des/der Beschäftigten so darstellte, dass er/sie nach seiner/ihrer Ausbildung lebenslang täglich in einen Betrieb zur Arbeit geht, dort eine bestimmte Arbeitszeit verbringt und, wenn es halbwegs passt, in dem Betrieb eine bestimmte vertikale Karriere durchläuft. Diese Form der Normalbiographie eines/r Beschäftigten trifft heute jedoch nur mehr eingeschränkt zu. Veränderungen wie verstärktes Telecommuting, vermehrt Arbeit in zeitlich begrenzten Arbeitsteams bis hin zu Arbeitsverhältnissen, die immer mehr zu zeitlich begrenzten Arbeitsbeziehungen zu Unternehmen führen, machen den Arbeitsalltag aus. Dazu kommt die schon oben beschriebene zunehmende Bereitschaft, auf die Suche nach guten, sinnerfüllten neuen Jobs zu gehen. Diese Art der Ausgestaltung von Arbeitsbeziehungen findet sich in bestimmten Feldern wie dem künstlerischen Bereich oder dem Profisport schon länger, nunmehr auch in immer größerer Zahl in bürokratischen, hierarchischen Organisationen, die trotz aller modernen Organisationsformen, wie Hypertext-, Cluster- oder anderen Formen von Netzwerkorganisationen, noch immer die Grundprinzipien vieler Organisationen ausmachen.

Die Ausdifferenzierungen der sich daraus ergebenden neuen Beschäftigungsformen lassen sich auf unterschiedliche Art und Weise systematisieren: Es wiederholen sich dabei hier die arbeitsrechtliche Verankerung in Form eines Vertrages, die Regelung von Kontrolle bzw. Weisungsgebundenheit, Arbeitszeit, -dauer oder aber auch Arbeitsort. Bei der Ausdifferenzierung dieser Kriterien gibt es bei den 
Abb. 1 Dimensionen der Beschäftigungsflexibilisierung

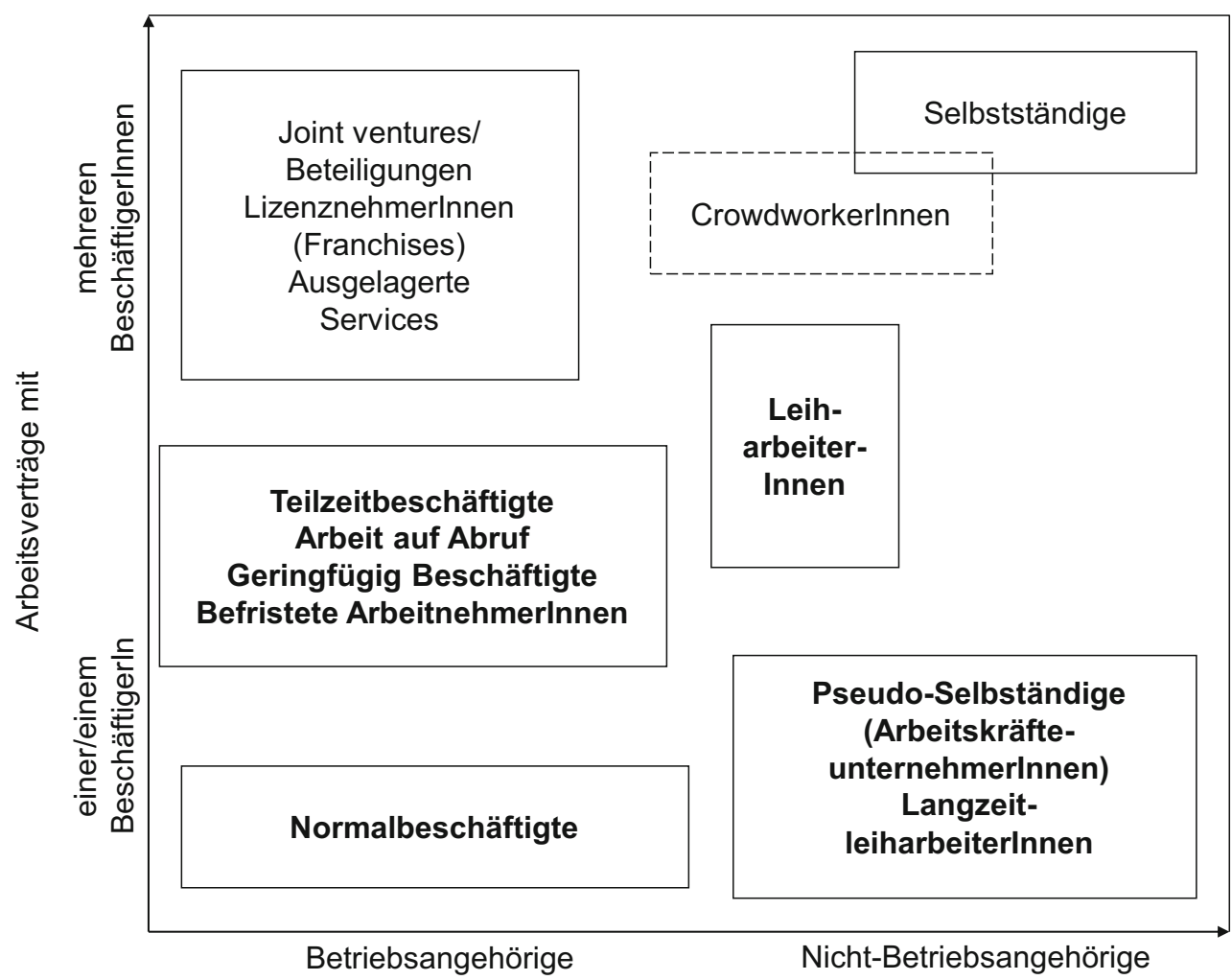

heute weit verbreiteten Beschäftigungsformen ganz unterschiedliche Kombinationen, die in der Folge entlang einer Systematik, die Grimshaw et al. (2005, S. $17 \mathrm{ff}$ ) entwickelt haben, analysiert werden. In dieser Systematik werden auf der einen Seite (x-Achse) das Zugrundliegen eines ,normalen" Arbeitsvertrages von neuen Formen unterschieden. Die zweite Dimension zielt auf die Anzahl der Beschäftiger_innen ab (siehe Abb. 1).

Räumliche und zeitliche Entgrenzungen gehen mittlerweile zunehmend mit rechtlicher Flexibilisierung einher und bewirken eine Veränderung der Einbettung von Arbeitsbeziehungen und -praktiken. Schon bei den Regelungen des Arbeitsorts finden sich Abweichungen von der Normalbeschäftigung. Die häufigsten und vielfältigsten Abweichungen von der Normalbeschäftigung finden sich jedoch in der zeitlichen Flexibilisierungsdimension. Insgesamt lässt sich neben der Flexibilisierung von Umfang, Lage und Dauer der Arbeitszeit auch die Abnahme von langandauernden bzw. dauerhaften Verträgen beobachten.

Hier sind es nicht nur Selbstständige und Lizenznehmer_innen, die ihren Arbeitsort und -zeit selbst definieren, sondern auch die Gruppe der Leiharbeiter_innen findet insbesondere ihren Arbeitsort nicht bei dem/der Beschäftiger_in. Nochmals anders gelagert ist diese Entgrenzung bei Crowdworker_innen, deren Aufträge über intermediär wirkende Online-Plattformen vermittelt werden. Die Zuordnung dieser letztgenannten Gruppe ist gar nicht leicht, da es hier sehr viele unterschiedliche Ausprägungen gibt
(Leinmeister 2016). Außerdem lassen sich in dieser Systematik auch Arbeitnehmer_innen in arbeitsmarktpolitischen Maßnahmen (dem zweiten Arbeitsmarkt: Kromphardt und Scheidt 1994) nicht wirklich zuordnen, da hier die $\mathrm{Zu}$ ordnung bzgl. der Arbeitsverträge bzw. der Zugehörigkeit mehrschichtig sein könnte. Ähnliches gilt auch für Beschäftigte, die unter die sogenannte informelle Ökonomie (Frambach 1999) oder Konzepte wie die Bürgerarbeit (Beck 1999) fallen. Unter diesen Sektor fallen Eigenarbeit, illegale Beschäftigung, Schwarzarbeit oder auch Nachbarschaftshilfe.

Eine wichtige Voraussetzung für diese Entwicklung war und sind die Entwicklungen im Bereich der Informationstechnologien, die die räumlichen, zeitlichen, aber auch rechtlichen Dimensionen von Beschäftigungsverhältnissen mit beeinflusst haben und beeinflussen. Dabei ist ein Aspekt zentral: Es scheint nicht nur so, dass die technologischen Veränderungen die Arbeit bzw. das Arbeitsverhalten mediieren, sondern sie vollziehen organisationale Realitäten und nehmen somit einen besonderen Stellenwert ein. Ausgangspunkt der weiteren Argumentation ist die Analyse der Beweggründe von Unternehmen für die Einführung bzw. Umsetzung von immer stärker ausdifferenzierten Beschäftigungsstrategien. 


\section{Von der Vermarktlichung über die Entgrenzung zum Identitätswandel}

Beim Blick auf die Arbeitsmärkte (Makroebene) sind es mittlerweile weniger hierarchische Strukturen, die als Steuerungsstrukturen wirken. Vielmehr wirken nunmehr Marktmechanismen bis hin zur Ausbildung von Netzwerkstrukturen mit allen Ausprägungen wie auch Schräglagen, die hier durch asymmetrische Beziehungen entstehen können. Darunter fallen beispielsweise Formen des Dependent Outsourcing - also z. B. Leiharbeit oder Crowdworking, denn in diesen Fällen liegt die Definitionsmacht über die Inhalte der Tätigkeiten bei den Auftraggebern.

Die in der Folge verschieden ausgestalteten Spielräume für die Akteure am Arbeitsmarkt sind ein wichtiger Faktor, warum sich Arbeitsbedingungen in Organisationen polarisieren (Wagner 2001). Es lösen sich Arbeitsstandards an den Rändern des Arbeitsmarkts auf.

Auf organisationaler Ebene (Mesoebene) wiederum liegt ein zentraler Bezugspunkt zu den Arbeitsmarktstrukturen in der Ausgestaltung der jeweiligen Beschäftigungspolitik. Moderne Organisationen verändern ihre Strukturen immer öfter von bürokratischen Formen hin zu organischen, komplexen bzw. dynamischen Formen wie z. B. netzwerkähnliche Formen. Hier lässt sich eine Ausdifferenzierung dieser Politiken beobachten. In der folgenden Übersicht (Tab. 1 in Anlehnung Mühlberger 2007, S. 95) werden die wichtigsten Merkmale zusammengefasst, die sich im Hinblick auf diese verschiedenen Steuerungsstrukturen von Beschäftigungssystemen unterscheiden lassen.

Bei diesem Überblick werden klassischen Steuerungsstrukturen wie dem Markt und der Hierarchie Netzwerkformen, die Powell (1990, S. 300) identifiziert hat, und der Form der unfreiwilligen Auslagerung (Dependent Outsourcing), die von Mühlberger (2007) geprägt wurde, gegenübergestellt.

Hierarchien repräsentieren die klassische Steuerungsfunktion in Unternehmen, wobei die normative Ausgangsbasis die Sichtweise eines Herrschaftsverhältnisses ist und damit der Spielraum für den/die Beschäftigte als sehr niedrig eingestuft werden kann und dies mit einem hohen Ausmaß an Abhängigkeit von dem/der Beschäftiger_in einhergeht. Diese Abhängigkeit hat auch noch eine andere Seite. Das Angebot war und ist in solchen Organisationen sehr oft Arbeitsplatzsicherheit und eine Karriereperspektive. Es gibt eine Unzahl an empirischen Studien, die aufzeigen, dass z. B. die Abstimmung der wechselseitigen Erwartungen und damit des Abschlusses eine gemeinsamen psychologischen Vertrags (Rousseau 2001) positive Ergebnisse von steigender Arbeitszufriedenheit über höheres organisationales Commitment bis hin zu erhöhter Performance begünstigen.
Marktnähere Steuerungsprinzipien, wie eine Vielzahl der neueren Vertragsformen repräsentieren, gehen von einer stärker vertragsorientierten Sichtweise der Verfügungsrechte aus. In dieser Orientierung an der Ausgestaltung des Vertrags als Grundlage liegen höhere Flexibilisierungsund niedrigere Abhängigkeitsgrade für den/die Beschäftigte, wobei es in dieser Hinsicht auch Unterschiede zwischen den einzelnen Formen gibt. Diese Form kommt den eingangs angeführten Haltungen Arbeit gegenüber um einiges mehr entgegen, als die traditionellen hierarchischen Strukturen.

Durch die Betrachtung von Organisationsprozessen als Wertketten und der damit einhergehenden Unterscheidung von Kern- und Supportprozessen sind die Voraussetzungen für Ausgliederungen geschaffen und somit auch ein wesentlicher Schritt hin zur Ausbildung von netzwerkähnlichen Organisationsformen getätigt. „If there is anything 'new' about the 'network' form, it is probably the increase in (reengineered) cross-functional, temporary project working within organizations and the decomposition of vertically integrated organizations as activities are outsourced to specialist providers or shared through collaborative arrangements" (Grimshaw et al. 2005, S. 14f). Netzwerkähnliche Formen passen sehr gut zu den ressourcenbasierten Ansätzen, vorausgesetzt, dass Manager_innen zwischen verschiedenen Formen der Externalisierung unterscheiden und die Vorteile von vertrauensintensiven Beschäftigungssystemen auch auf externe Beziehungen ausgeweitet werden. Im Hinblick auf die Ausprägungen der Steuerungsstrukturen wird gerade bei dieser Form ersichtlich, wo die Chancen aber auch die Risiken liegen: es geht um ein komplementäres Verhältnis der beteiligten Partner_innen und ist durch die gegenseitige Abhängigkeit geprägt. Darin liegt ein Potential für Synergien, die sich aus dieser komplementären Beziehung ergeben können, aber auch möglichen Risiken, die sich aus dieser gegenseitigen Abhängigkeit ergeben können.

Damit verbunden sind auf der Steuerungsebene in den Organisationen unterschiedliche Spielräume. Es verändert sich der Zugang von hohen Steuerungsmöglichkeiten in Normalbeschäftigungen hin zu wenig Steuerungsmöglichkeiten und eingeschränkten Kontrollmechanismen. Im Bereich der Normalbeschäftigung, die sich in zumeist hierarchisch organisierten Unternehmen finden, gibt es bzgl. der Steuerung und Kontrolle ein Set an Regeln und Routinen. In nicht-hierarchischen Bereichen werden diese zunehmend durch Preise ersetzt und in Netzwerken wiederum passiert Steuerung durch Ausgestaltungen von Beziehungen. Wobei die Dimension der Ausprägung der Beziehungen noch um die Ausprägung der Kopplung zu erweitern ist. Es zeigt sich dabei, dass sich der Grad der Koppelung immer stärker lockert und sich als weniger stark ausgeprägt darstellt. 
Tab. 1 Entwicklung der Steuerungsstrukturen innerhalb von Beschäftigungssystemen

\begin{tabular}{|c|c|c|c|c|c|}
\hline & & \multicolumn{4}{|l|}{ Steuerungsstruktur } \\
\hline & & Hierarchie & Markt & $\begin{array}{l}\text { Netzwerk/ } \\
\text { Relationaler Markt }\end{array}$ & $\begin{array}{l}\text { Dependent } \\
\text { Outsourcing }\end{array}$ \\
\hline \multirow[t]{4}{*}{$\overline{\text { Merkmale }}$} & Normative Basis & $\begin{array}{l}\text { Beschäftigungsverhältnis } \\
\text { Herrschaft }\end{array}$ & $\begin{array}{l}\text { Vertraglich fixierte } \\
\text { Eigentumsrechte } \\
\text { Lieferbeziehung, } \\
\text {-vertrag }\end{array}$ & $\begin{array}{l}\text { Komplementäre } \\
\text { Stärken }\end{array}$ & $\begin{array}{l}\text { Formale und relatio- } \\
\text { nale Verträge }\end{array}$ \\
\hline & $\begin{array}{l}\text { Ausmaß der Flexi- } \\
\text { bilität für den/die } \\
\text { Beschäftiger/in }\end{array}$ & Niedrig & Hoch & Mittel bis hoch & Mittel \\
\hline & $\begin{array}{l}\text { Ausmaß der Abhän- } \\
\text { gigkeit des/der Be- } \\
\text { schäftigers/in }\end{array}$ & Abhängig & Unbhängig & Gegenseitig abhängig & Abhängig \\
\hline & Mischformen & $\begin{array}{l}\text { Marktähnliche Fea- } \\
\text { tures: Profit centres, } \\
\text { transfer pricing }\end{array}$ & $\begin{array}{l}\text { Vertrag als hierarchi- } \\
\text { sierendes Dokument }\end{array}$ & $\begin{array}{l}\text { Mehrere PartnerIn- } \\
\text { nen formale Regeln }\end{array}$ & - \\
\hline
\end{tabular}

Mit diesen Flexibilisierungen und damit auch immer stärker individualisierten Arbeitsarrangements gehen Fragen des Zugriffs und der Kontrolle von (formal) ,außerhalb“ des Unternehmens angesiedelten „Humanressourcen“ bis hin zu motivationalen und loyalitätsorientierten Fragestellungen einher.

Veränderte Arbeitszeiten und -rythmen und veränderte Grundhaltungen, die ausdifferenzierte Steuerungssysteme mit sich bringen, wirken auf die Führungsbeziehungen mit ein. Damit verändert sich der Stellenwert physischer Anwesenheit bzw. der persönliche Kontakt, wobei beides als ein Element der Kontrolle auch in neueren Debatten angesprochen wird.

Kratzer (2002) spricht davon, dass eine entscheidende Veränderung im Modus der Steuerung und Kontrolle von Arbeit liegt. Dieser Modus hat sich in einer Art indirekten Steuerung verändert: Das Management überlässt wesentliche Aspekte der Steuerung und Organisation der Arbeit den Beschäftigten selbst, legt aber zugleich Ziele und den weiteren Rahmen fest und kontrolliert die Zielerreichung. Dabei wird sehr oft von Selbstorganisation gesprochen. Dies ist nicht als Ende der Kontrolle zu sehen, sondern vielmehr als Umbruch im Modus der Kontrolle und Steuerung von Arbeit, der sich dadurch auszeichnet, dass sich Herrschaft gerade durch Autonomie realisiert (Moldaschl und Sauer 2000). Wir finden also immer stärker individualisierten Arbeitsarrangements vor. Autonomie und Kontrolle stehen an sich schon in einer widersprüchlichen Beziehung - das haben Frost und Osterloh (2002) schon sehr ausführlich dargestellt. Durch räumliche und zeitliche Entgrenzungen entstehen nochmals mehr neue Facetten, wie diese an sich Koexistenz gelebt werden können. In diesem Zusammenhang zeigen Bader und Kaiser (2017), dass die vermeintlichen, individuellen Gestaltungsräume, die sich durch neue Regelungen und bedingt durch neue Technologien ergeben haben, stark durch kollektive Normen beschränkt werden und sie sehen einen Trend zu einer vorherrschenden Rolle von Kontrolle. An der Stelle sei an die aufkommende, kritische Diskussion von algorithmischem Management verwiesen, im Rahmen zunehmend mehr Bereiche des organisatorischen Handelns datengestützt gesteuert werden - und entsprechend auch für Rechenschafts- und Kontrollprozesse eingesetzt werden (Luca et al. 2016; Beverungen 2017).

Neben den Auswirkungen auf Steuerung und Kontrolle der Arbeit bringen neue Beschäftigungsformen mit sich, dass Unternehmen die gewohnten identifikationsstiftenden Symbole wie einen eigenen Arbeitsplatz nicht mehr in dieser Form bereitstellen (Linde 1997, S. 22). Es verschieben sich die Symbole. Veränderte Steuerungsformen gehen auch mit einer Veränderung formaler Strukturen einher. Den Trend hin $\mathrm{zu}$ organischen, komplexen bzw. dynamischen Formen wie z. B. netzwerkähnliche Formen wurde oben schon beschrieben. Das führt nach Ansicht von Ashforth und Johnson (2001, S. 36) dazu, dass somit ,lower order identities such as the workgroup“ für den/die einzelne/n Beschäftigte wichtiger werden als z. B. Symbole wie ein eigenes Büro. Dies unterstreicht auch Dale (2005, S. 660): ,changing social and economic conditions require changing forms of control incorporated into different spatial and material arrangements."

Als solche möglichen Formen wird in weiterer Folge auf zwei wichtige mögliche Arrangements verwiesen: Management of Meaning and Management of Identity. Diese Formen unterstützen die Kontrollidee, die sehr stark mit traditionellen, vertikalen und hierarchischen Formen der $\mathrm{Au}$ toritätsbeziehungen verbunden ist, stärker im Sinne von horizontalen Formen wie Peer- und Teamkontrollen zu sehen. Die horizontalen Formen gehen vom konzeptionellen Gedanken her stark mit Selbstdiziplinierung bzw. „Alignment of the Self with the Company“ (Dale 2005) einher und sie zeigen sich in einer Kombination von kulturellen und textlichen Interventionen, die stark wirkende Werte widerspie- 
geln und als Basis für individuelles Commitment fungieren. Es gibt somit eine Verbindung zwischen dem Thema Kontrolle, Kontrollformen und Bindungsformen. In diese Richtung geht die Überlegung, dass bei Beschäftigte, die physisch, zeitlich und administrativ losgelöst von den Organisationen arbeiten, Identitätsbezugspunkte vor allem in der Arbeit selbst und weniger im organisationalen Geschehen: ,Specifically, they find that independent workers make meaning by drawing on their self-knowledge and culturally available meaning units rather than through the interaction with others" (Ashford et al. 2007, S. 72). Dieser Mechanismus der Bedeutungszuschreibung an Arbeit und in weiterer Folge an Identitätsbildung resultiert aus den veränderten Arbeitsbedingungen und hat Rückwirkungen auf den Bereich der Formen der Einflussnahme.

Somit ist neben dem Thema Kontrolle und Einflussnahme auch die Selbstsicht der Beschäftigten im Hinblick auf das Verhalten ein essentielles Thema und es gilt, den Focus auf die Form des abgeschlossenen psychologischen Vertrags und der Koppelung des/der Einzelnen zu richten.

\section{Koppelung und Identifikationsprozesse}

Eine Tätigkeit geht mit einer Vereinbarung und somit in den überwiegenden Fällen konkret mit einem Arbeitsvertrag einher. Durch die im (Arbeits)Vertrag gestalteten, unterschiedliche Ausprägungen im Hinblick auf z. B. Arbeitsort oder Zeit werden lose und enge Koppelung gerade im Hinblick auf die Ausbildung von Nähe und Distanz des/der Einzelnen zum Unternehmen (zur Organisation) beeinflusst. Arbeitsverträge, denen Normalbeschäftigungen zugrunde liegen, laufen aufgrund z. B. der damit verbundenen Zeitwahrnehmung oder auch der Zuordnung von Aktivitäten auf eine enge Koppelung hinaus, während atypische Formen mit einer loseren Koppelung einhergehen.

Die Ausprägungen loser bzw. enger Koppelungen spielen insbesondere hinsichtlich der Erhaltung bzw. Bewahrung der Überlebensfähigkeit eines Systems eine wichtige Rolle. Dabei sind vor allem kontextuellen Bedingungen nicht zu vernachlässigen.

Einer der essentiellen Kontextfaktoren, die auf die Grenzziehung und damit auch auf die Ausgestaltung von Mitgliedschaftsrollen wirken, sind Zeit und Raum. Mitgliedschaft manifestiert sich auch immer in Bezug auf die Mitwirkung an Entscheidungen und konstituiert sich gerade dadurch als kommunikative Errungenschaft immer wieder neu. Ein beispielhafter Fall dafür ist die Sitzung, auf der etwas beschlossen werden soll. Dabei zeigt sich, dass eine nicht jederzeit durch Mitgliedschaft begründete Anwesenheit Gefahr läuft, kommunikativ auffällig und sanktioniert $\mathrm{zu}$ werden. Akte von Reglementierungen sind in dem an- geführten Beispiel die Wahl von Ab- und Anwesenheit, die Platzierung im jeweiligen Raum und das Ausnutzen situativer Ressourcen, wie z. B. die Organisation und das Recht zu reden und zu schweigen, die Wahl der Themen und die Kontextualisierung dieser Inhalte und ähnliches. Diese Reglementier- und Formalisierungen verweisen auf Entscheidungen als jederzeit greifbares und jederzeit relevantes Letztelement der Kommunikation.

Wenn nun atypische Beschäftigungsformen Relativierungen von ortsfesten Arbeitsformen bzw. Verwischungen der zeitlichen Normvorstellungen mit sich bringen, verändern sich mit diesen Entstandardisierungen die Möglichkeiten und Anforderungen, wie Mitgliedschaft kommunikativ hervorgebracht werden kann. Damit ist gemeint, dass sich die sachlichen, zeitlichen und sozialen Bezüge verändern. So verändert sich $\mathrm{z}$. B. durch nicht ständige Anwesenheit die Informationsgrundlage bzw. wird durch Befristungen Verbundenheit auch anderes gesehen. Diese Wirkung der Veränderung der Mitgliedschaftsrolle zeigt sich auf der Mikroebene besonders in der Ausgestaltung der Arbeitsbeziehungen. Dabei treten folgende zentrale Fragen ins Blickfeld: wie wirken die Veränderungen auf Leistungssteuerungssysteme und damit auch die Beziehung von Führenden und Geführten in Organisationen und wie verändern sich Erwartungslagen von Beschäftigten?

Durch die Ausdifferenzierung von Beschäftigungsformen werden die bisher für Normalbeschäftigung definierten Formen der Beziehungsgestaltung, die sich in der physischen Nähe, dem Grad der administrativen Kontrolle und der Dauer der erwarteten Beschäftigung manifestieren, auf den Prüfstand gestellt. Alle diese drei Formen beziehen sich auf die Normalbeschäftigung, die vor Ort in der Organisation erbracht wird. Wenn die erwartete Beschäftigungsdauer begrenzt ist, schränkt sich die Spannweite der administrativen Kontrolle ein - ähnliches wird für die physische Nähe angeführt. Auch hier - bei annehmender physischer Nähe - wird von einem Kontrollverlust ausgegangen, der zunehmend durch andere Formen, wie z. B. datenbasierter Kontrollen ersetzt werden.

Es finden sich bei dem/der einzelnen unterschiedliche Selbstbestimmungsgrade ebenso wieder wie veränderte Formen von psychologischen Verträgen. Subjektivierungen prägen hier die Diskussion und haben Auswirkungen auf Identifikationsprozesse. Der Bezug auf Gruppennormen bleibt zwar weiterhin ein wichtiger Aspekt, wobei es bei neuen Beschäftigungsformen weniger die Bezugsgruppe in der Organisation ist, als Bezugsgruppen, die außerhalb der Organisationen angesiedelt sind (Berufsgruppen, Netzwerke). Diese Verschiebungen haben auch Wirkungen auf die Haltungen (,mind sets") der Mitglieder in Organisationen und beeinflussen die Ausbildung der Identitätsgrenzen in und von Organisationen. Identitätskonstruktionen von dem/der einzelnen Beschäftigten sind somit ebenso 
wesentlich für die Ausbildung eines psychologischen Vertrags, wie die Frage, wie situationsabhängige, subjektive Einschätzung von Versprechen und Verpflichtungen angesehen wird. Die Einstellung zur Beschäftigungssicherheit oder auch die erlebte Work-Life-Balance beeinflussen den Status des psychologischen Vertrags und wirken somit auf verschiedene beobachtbare und z.T. messbare Ergebnisse der Arbeitsbeziehung, wie die Arbeitszufriedenheit, der Abwesenheit, der Intention des Jobwechsels und eben auch der Ausprägung des Engagements und dem organisationalen Commitments.

Es gibt aber auch entsprechend zentrale Kontextfaktoren, die sich schon in der Darlegung der Veränderungen zuvor deutlich wurden. Zeit, erlebter Handlungsspielraum und soziale Gerechtigkeit werden hier nochmals im Hinblick auf deren Wirkung auf Bindung hervorgehoben: Vom Faktor Zeit haben wir schon mehrfach gehört. An der Stelle soll besonders darauf hingewiesen werden, dass die Analyse der Ausdifferenzierung von Beschäftigungsverhältnissen ergeben hat, dass Zeit im Sinne von Arbeitszeit bzw. Vertragsdauer eines der Hauptunterscheidungskriterien ist. Die $\mathrm{Zu}-$ schreibungen und deren Veränderung über die Zeit werden als wesentlicher Treiber gesehen, der die Einschätzung von Vertragserfüllungen oder die Einschätzung der Stärke der Bindungskraft beeinflussen (Ho 2005). Ein weiterer Faktor bezieht sich auf den erlebten Handlungsspielraum. Es geht in diesem Zusammenhang um die Möglichkeiten, Aufgaben und Handlungsspielräume als gestaltbar einzuschätzen (Ng et al. 2006). Diese subjektiv wahrgenommenen Spielräume werden stark durch Umweltfaktoren wie organisationale Kontexte in Form der Human Ressource Politiken oder auch die Organisationskultur geprägt. Es wirken hier aber auch andere Umweltfaktoren wie die Form der sozialen Einbettung mithinein. Verbunden ist dieser Faktor auch mit Überlegungen, wie soziale Gerechtigkeit erlebt wird bzw. wie mit Unsicherheit umgegangen wird. So kann Gerechtigkeit selbst als ein Ziel des Commitments von dem/der Einzelnen gesehen werden (Boxall und Purcell 2003). Damit wird die verschieden erlebte Gerechtigkeit zu einem Bezugspunkt für Commitment. In der Interaktionsgerechtigkeit liegt ein weiterer Anknüpfungspunkt, denn die erlebte Vorgangsweise und die jeweiligen zugrundeliegenden Interaktionen können als zentrale Referenzpunkte für die erlebte Verbundenheit gelten. Allen genannten Faktoren ist gemein, dass sie bis jetzt in den Konzeptionen von organisationalem Commitment nur teilweise mitberücksichtigt wurden und als Anstoß für die Reformulierung der Commitmentkonzepte dienen können (Schmidt 2013).

\section{Conclusio}

Die aufgezeigten Faktoren bergen alle das Potenzial in sich, sich als Trenn- bzw. Verwerfungslinien (faultlines) in Organisationen darzustellen. „Faultlines may act like simmering pots that are not yet boiling over, but if the heat (or tension) is increased, they would begin to boil." (Hannum et al. 2010, S. 14) Durch Anstöße, wie z. B. unterschiedliche Behandlung, können diese Trennlinien aktiviert und „zum Kochen gebracht“ - also ähnlich einem Vulkan zum Ausbruch gebracht - werden. Damit werden sie innerhalb von Organisationen als Konfliktlinien relevant. Dass diese Verwerfungslinien auch schon in den Unternehmen erkannt werden, zeigen aktuelle Studienergebnisse. Jedes dritte Jahr führt die Boston Consulting Group eine Erhebung bei Personalverantwortlichen durch, um die aktuellen Trends im Bereich der Personalpolitik auszuloten. Wenn wir uns die Topthemen 2008 (kurz vor der Finanzkrise) betrachten, ist das Topthema Talentmanagement, gefolgt von Leadership Development, Optimierung der Recruitierung und Performance Management. Schaut wir uns die letzten zugänglichen Ergebnisse aus dem Jahr 2015 an, so bleibt Talentmanagement ein Dauerbrennerthema. Die nachfolgenden Toptrends haben jedoch einen ganz anderen Fokus: Dort finden sich On-Boarding und Retention-Management, Demographie-Management und das Management von WLB (Strack et al. 2011, 2016) - und hier schließt sich der Kreis zu in der Einleitung dargelegten Motiven der Beschäftigten.

Funding Open access funding provided by Vienna University of Economics and Business (WU).

Open Access Dieser Artikel wird unter der Creative Commons Namensnennung 4.0 International Lizenz (http://creativecommons.org/ licenses/by/4.0/deed.de) veröffentlicht, welche die Nutzung, Vervielfältigung, Bearbeitung, Verbreitung und Wiedergabe in jeglichem Medium und Format erlaubt, sofern Sie den/die ursprünglichen Autor(en) und die Quelle ordnungsgemäß nennen, einen Link zur Creative Commons Lizenz beifügen und angeben, ob Änderungen vorgenommen wurden.

\section{Literatur}

Albert, S., Ashforth, B.E., \& Dutton, J.E. (2000). Organizational identity and identification: Charting new waters and building new bridges. Academy of Management Review, 25(1), 13-17.

Appelbaum, E., Bailey, T., Berg, P., \& Kalleberg, A. (2005). Manufacturing advantage: Why high-performance work systems pay off. Ithaca: Cornell University Press.

Ashforth, B., \& Johnson, S. (2001). What hat to wear? The relative salience of multiple identities in organizational contexts. In M. Hogg \& d. Terry (Hrsg.), Social identity processes in organizations (31-44). New York: Pschology Press.

Ashford, S. J., George, E., \& Blatt, R. (2007). Old assumptions, new work. In J.P. Walsh \& A.P. Brief (Hrsg.), The academy of management annals (Bd. 1, S. 65-117). London: Routledge. 
Bader, V., \& Kaiser, St (2017). Autonomy and control? How heterogeneous sociomaterial assemblages explain paradoxical rationalities in the digital workplace. Management revue, 28(3), 338-358.

Beck, U. (1999). Schöne neue Arbeitswelt. Frankfurt: Suhrkamp. Vision: Weltbürgerschaft

Beverungen, A. (2017). Algorithmisches Management. In T. Beyes, J. Metelmann \& C. Pias (Hrsg.), Nach der Revolution, Ein Brevier digitaler Kultur (S. 52-63). Hamburg: Edition Speersort.

Boxall, P., \& Purcell, J. (2003). Strategy and human resource management. New York: Palgrave.

Dale, K. (2005). Building a social materiality: Spatial and embodied politics in organizational control. Organization, 12(5), 649-678.

Frambach, H. A. (1999). Zum Verständnis von Arbeit im historischen Wandel. Eine Untersuchung aus nationalökonomischer Perspektive. Arbeit, 11(3), 226-243.

Frost, J., \& Osterloh, M. (2002). Motivation und Organisationsstrukturen. In M. Osterloh \& B. Frey (Hrsg.), Managing Motivation. Wie Sie die neue Motivationsforschung für Ihr Unternehmen nutzen können (2. Aufl. S. 166-192). Wiesbaden: Gabler.

Gallup (2017). Engagementindex - Pressegespräch. http://www. gallup.de/183104/engagement-index-deutschland.aspx. Zugegriffen: 27. Juni 2018.

Grimshaw, D., Marchington, M., Rubery, J., \& Willmott, H. (2005). Introduction: fragmenting work across organizational boundaries. In M. Marchington, D. Grimshaw, J. Rubery \& H. Willmott (Hrsg.), Fragmenting work: Blurring organizational boundaries and disordering hierarchies (S. 1-38). New York: Oxford University Press.

Hannum, K., McFeeters, B. B., \& Booysen, L. (2010). Leading across differences. San Francisco: Wiley.

Harley, B. (2005). Hope or hype? High-performance work systems. In D. Knights, P. Thompson, C. Smith \& H. Willmott (Hrsg.), Participation and democracy at work. Essays in honour of Harvie Ramsay (S. 38-54). Houndsville: Palgrave.

Ho, V.T. (2005). Social Influence on evaluations of psychological contract fulfillment. Academy of Management Review, 30(1), $113-128$.

Kratzer, N. (2002). Warum machen die Beschäftigen mit? Erklärungsansätze für die Ambivalenz selbstorganisierter Arbeit. http:// www.isf-muenchen.de/pdf/Cogitonk.pdf Vortragsmanuskript für die Tagung „Unselbständig Selbständige oder Arbeitskräfteunternehmer", Mai 2002 in Köln.

Kromphardt, J., \& Scheidt, B. (1994). Chancen des „Zweiten Arbeitsmarktes". Wirtschaftsdienst, 12, 615-622.

Lee, T. W., Hom, P., Eberly, M., \& Li, J. J. (2018). Managing employee retention and turnover with $21^{\text {st }}$ century ideas. Organizational Dynamics, 47, 88-98.

Leinmeister, J. M., Durward, D., \& Shkodran, Z. (2016). Crowd Worker in Deutschland. Düsseldorf: Edition Hans Böckler Stiftung.

Linde, F. (1997). Neue Kooperationsformen auf dem Prüfstand: Virtuell kann nicht völlig ,grenzenlos‘ bedeuten. Gablers Magazin, 13, 20-23.

Luca, M., Kleinberg, J., \& Mullainathan, S. (2016). Algorithms need managers, too. https://hbr.org/2016/01/algorithms-needmanagers-too Harvard Business Review, Jan-Feb, 98-101.
Luthans, F., \& Youssef, C. M. (2004). Human, social, and now positive psychological capital management: Investing in people for competitive advantage. Organizational Dynamics, 33(2), 143-160.

Moldaschl, M., \& Sauer, D. (2000). Internalisierung des Marktes - Zur neuen Dialektik von Kooperation und Herrschaft. In H. Minssen (Hrsg.), Begrenzte Entgrenzungen (S. 205-224). Berlin: Ed. Sigma.

Mühlberger, U. (2007). Dependent self-employment. Workers on the border between employment and self-employment. London: Palgrave Macmillan.

Ng, T. W.H., Sorensen, K. L., \& Eby, L. T. (2006). Locus of control at work: A Meta-analysis. Journal of Organizational Behavior, 27, 1057-1087.

Oldham, G. R., \& Hackman, J. R. (2010). Future of job design research. Journal of Organizational Behavior, 31, 463-479.

Powell, W.W. (1990). Neither market nor hierarchy: Network forms of organization. In B. M. Staw \& L. L. Cummings (Hrsg.), Research in organizational behavior (Bd. 12, S. 295-336). London: Jai Press.

Rousseau, D. (2001). The Ideosyncratic Deal: Flexbility versus Fairness? Organizational Dynamics, 29(4), 260-273.

Schmidt, A. (2013). Organisationales Commitment und Beschäftigungsverhältnisse. Eine Operationalisierung unter dem Aspekt sich verändernder Beschäftigungsverhältnisse. Frankfurt am Main: Peter Lang.

Sharkie, R. (2005). Precariousness under the new psychological contract: The effect on trust and the willingness to converse and share knowledge. Knowledge Management Research \& Practice, 3, 37-44.

Strack, R., Haen, P., Caye, J. M., Frick, G., Teichmann, C., \& Bird, S. (2011). Creating people advantage 2008. Time to act: HR certainties in uncertain times. Boston: The Boston Consulting Group.

Strack, R., Caye, J.M., Leicht, M., Villis, U., Böhm, H., \& McDonnell (2016). Creating people advantage 2015. The future of HR in europe. Boston: The Boston Consulting Group.

Wagner, A. (2001). Entgrenzung der Arbeit und der Arbeitszeit? Arbeit, 10(3), 365-378.

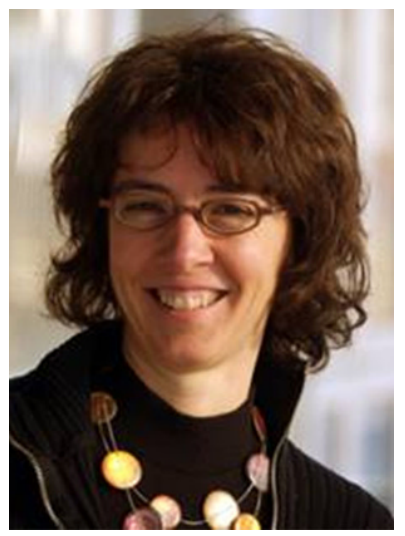

Angelika Schmidt Dozentin am Institut für Change Management und Management Development der WU Wien. Studium der Wirtschaftspädagogik und Volkswirtschaft an der WU Wien. Forschungsschwerpunkte: Schnittstellen in Organisationen (z. B. Beruf/Privat, Geschlechterfragen, Generationen), neue Beschäftigungsformen und deren Konsequenzen und organisationale Diskurse. 